\title{
TEMPO DE VIABILIDADE DE AMOSTRAS DE SANGUE VENOSO BOVINO DESTINADAS AO EXAME HEMOGASOMÉTRICO, QUANDO MANTIDAS SOB CONSERVAÇÃO EM ÁGUA GELADA
}

\author{
VIABILITY TIME OF BLOOD GAS ANALYSIS IN BOVINE VENOUS BLOOD SAMPLES \\ STORED IN ICE WATER BATH
}

\author{
Júlio Augusto Naylor Lisbôa ${ }^{1}$ Fernando José Benesi ${ }^{2}$ Celso Akio Maruta ${ }^{3}$ \\ Regina Mieko Sakata Mirandola ${ }^{4}$ Cynthia Maria Carpigiani Teixeira $^{3}$
}

\section{RESUMO}

Com o objetivo de verificar o tempo de viabilidade de amostras de sangue venoso bovino destinadas ao exame hemogasométrico, quando conservadas em banho de água gelada, selecionaram-se 14 bovinos ( 7 machos e 7 fêmeas), clinicamente sadios, entre 1 e 5 anos de idade. Colheram-se, de cada animal, 2 amostras de sangue (10ml cada), por punção da veia jugular, com o emprego de seringas plásticas contendo cerca de 1.000UI de heparina sódica, tomando-se os cuidados de eliminar quaisquer bolhas de gás presentes, e de manter vedada a extremidade da agulha com rolha de borracha. Cada uma do par de seringas foi mantida, distintamente, à temperatura ambiente (entre 23 e $30^{\circ} \mathrm{C}$ ) ou submersa em água gelada (entre zero e $4^{\circ} \mathrm{C}$ ). As determinações do $\mathrm{pH}$, pressões parciais venosas de dióxido de carbono $\left(\mathrm{Pv}_{\mathrm{CO} 2}\right)$ e de oxigênio $\left(\mathrm{Pv}_{\mathrm{O} 2}\right)$, bicarbonato $\left(\mathrm{HCO}_{3}{ }^{-}\right)$, total de dióxido de carbono $\left(\mathrm{TCO}_{2}\right)$, excesso ou déficit de bases (BE), bicarbonato padrão $(\mathrm{StB})$, saturação de oxigênio $\left(\mathrm{SatO}_{2}\right)$ e do conteúdo de oxigênio $\left(\mathrm{O}_{2}\right)$ foram realizadas imediatamente e com 1, 2, 3, 4, 5, 6, 8, 10, 12 e 24 horas após a colheita. Em cada um dos critérios de manutenção do material, os resultados foram avaliados por meio da análise de variância de medidas repetidas. Consideradas as diferenças entre os valores médios obtidos em cada tempo e aquele da avaliação inicial, os resultados indicaram que as alterações "in vitro" das amostras não conservadas já eram marcantes a partir de 2 ou 3 horas, caracterizando-se por reduções contínuas do $\mathrm{pH}, \mathrm{Pv}_{\mathrm{O} 2}, \mathrm{BE}$, StB, Sat $\mathrm{O}_{2}$ e $\mathrm{O}_{2}$, e por elevação gradativa da $\mathrm{Pv}_{\mathrm{CO} 2}$. No sangue conservado, ao contrário, o valor de $\mathrm{pH}$ não exibiu diferenca do original durante as primeiras 4 horas, e os de $\mathrm{Pv}_{\mathrm{CO} 2}, \mathrm{BE}$ e StB mantiveram-se inalterados por até 6 horas após a colheita. A análise dos resultados permitiu concluir que as amostras de sangue venoso bovino destinadas ao exame de hemogasometria podem permanecer viáveis por até 6 horas, quando adequadamente conservadas em banho de água gelada, mantendo, assim, o seu valor diagnóstico.

Palavras-chave: Hemogasometria, conservação, sangue venoso, bovinos.

SUMMARY

In order to verify the viability of blood gas analysis in bovine venous blood stored on ice water bath, two samples (10ml each) were taken from the jugular vein of 14 healthy animals (7 males and 7 females), 1- to 5-year-old, using plastic syringes and attached needles filled with sodium heparin (1,000IU). The blood samples were obtained anaerobically, the air bubbles observed were immediately removed, and the needle was maintened capped with a rubber stopper. Each syringe of the pair was distinctally stored at room temperature $\left(23-30^{\circ} \mathrm{C}\right)$ or in ice water bath $\left(0-4^{\circ} \mathrm{C}\right)$ during the experimental period. Values of $\mathrm{pH}$, carbon dioxide $\left(\mathrm{Pv}_{\mathrm{CO} 2}\right)$ and oxigen $\left(\mathrm{Pv}_{\mathrm{O} 2}\right)$ tensions, bicarbonate $\left(\mathrm{HCO}_{3}^{-}\right)$, total carbon dioxide $\left(\mathrm{TCO}_{2}\right)$, base excess $(B E)$, standard bicarbonate (StB), oxigen saturation ( $\left.\mathrm{SatO}_{2}\right)$, and oxigen content $\left(\mathrm{O}_{2}\right)$ were determined soon after sampling and after 1, 2, 3, 4, 5, 6, 8, 10, 12 and 24 hours. According to the type of storage temperature, the results were analysed through repeated measurements ANOVA, considering the contrast between the mean value of each time and the initial one. On the storage at room temperature, the in vitro changes were characterized from continuous decreases in $\mathrm{pH}, \mathrm{Pv_{O2 }}, \mathrm{BE}, \mathrm{StB}$, $\mathrm{SatO}_{2}$, and $\mathrm{O}_{2}$ values, and gradual increase in $\mathrm{Pv}_{\mathrm{CO}_{2}}$ starting at 2or 3-hour after the collection. In the samples stored at $0-4^{\circ} \mathrm{C}$, on the other hand, the changes in $\mathrm{pH}$ occurred only at the $4^{\text {th }}$ hour, and the stability of the $P v_{\mathrm{CO} 2}, B E$, and $S t B$ values were maintened for up to the $6^{\text {th }}$ hour. These results indicated that the diagnostic

\footnotetext{
${ }^{1}$ Médico Veterinário, Professor Assistente, Mestre, Departamento de Clínicas Veterinárias. Universidade Estadual de Londrina, Campus Universitário, 86051-970, Londrina, PR. Aluno. Curso de Doutorado em Clínica Veterinária/ FMVZ/ USP. Autor para correspondência. ${ }^{2}$ Médico Veterinário, Livre-docente, Professor Associado, Departamento de Clínica Médica (VCM), Faculdade de Medicina Veterinária (FMVZ), Universidade de São Paulo (USP).

${ }^{3}$ Médico Veterinário, Aluno, Curso de Mestrado em Clínica Veterinária, FMVZ/USP.

${ }^{4}$ Farmacêutico Bioquímico, Mestre, Técnico de nível superior, VCM/FMVZ/USP.
} 
utility of blood gas analysis is conserved in bovine venous blood samples adequately stored up to 6 hours in ice water bath, at $0-4^{\circ} \mathrm{C}$.

Key words: blood gas analysis, storage, venous blood, cattle

\section{INTRODUÇÃO}

A avaliação dos gases sangüíneos e dos demais parâmetros necessários para a interpretação correta do equilíbrio ácido-básico oferece um conjunto de informações de grande valor para o prognóstico e decisão terapêutica de determinadas enfermidades dos bovinos. A importância dos resultados da hemogasometria pode ser destacada no caso de doenças de expressão econômica, tais como, a acidose ruminal aguda, as ectopias do abomaso e os processos toxêmicos em geral (BLOOD \& RADOSTITS, 1989), além das enteropatias com a manifestação de diarréia (GONÇALVES et $\boldsymbol{a l}$., 1991) e dos quadros de asfixia neonatal (BENESI, 1993). A necessidade de processar a amostra em menor tempo possível, desde a sua colheita (HASKINS, 1977; COLES, 1984), impõe-se, entretanto, como um sério fator de limitação, particularmente para o profissional que milita no campo.

A possibilidade de manutenção das amostras de sangue sob conservação a baixas temperaturas tem sido objeto de interesse de algumas investigações científicas nas espécies bovina (JAGOS et al., 1977; POULSEN \& SURYNEK, 1977; SZENCI \& BESSER, 1990; SZENCI et al., 1991), eqüina (ASSAL \& POULSEN, 1978; SZENCI et al., 1991), canina (HASKINS, 1977; ASSAL et al., 1978) e suína (ASSAL et al., 1980). As conclusões demonstram não haver, no entanto, um consenso a respeito do intervalo de tempo em que as amostras podem manter a sua viabilidade. $\mathrm{E}$ as indicações chegam a variar de 8 (JAGOS et al., 1977) a 24 horas (SZENCI \& BESSER, 1990) para o sangue venoso de bovinos.

Tendo em vista a importância de uma definição desse aspecto, o presente experimento foi delineado com o objetivo de verificar o tempo transcorrido desde a colheita, no qual amostras de sangue venoso bovino, conservadas em banho de água gelada, podem manter-se viáveis para a hemogasometria.

\section{MATERIAL E MÉTODOS}

\section{Colheita de material}

Selecionaram-se para o estudo 14 bovinos (7 machos e 7 fêmeas), clinicamente sadios, de diferentes raças e com idades variando entre 1 e 5 anos. Os experimentos foram realizados em dias programados, utilizando-se, no máximo, dois animais por vez, sendo, na véspera, obtido o material necessário para a determinação da hemoglobina. Para tanto, retiraram-se $5 \mathrm{ml}$ de sangue, por venopunção da jugular externa, empregando-se o sistema a vácuo ${ }^{\mathrm{a}}$ de tubo siliconizado com EDTA tripotássico (na proporção de $1,5 \mathrm{mg} / \mathrm{ml}$ de sangue) e agulha apropriada $25 \times 8 \mathrm{~mm}$.

As amostras usadas no experimento foram colhidas em duplicata, de cada animal, mediante punção da veia jugular externa, utilizando-se agulhas descartáveis $25 \times 8 \mathrm{~mm}$ acopladas a seringas plásticas de $10 \mathrm{ml}$ contendo, previamente, cerca de 1.000UI de heparina sódica ${ }^{\mathrm{b}}$. Para evitar o contato do sangue com os gases do ambiente, tomaram-se os cuidados rotineiros durante e após a colheita, consistindo em sucção lenta do material, eliminação de qualquer bolha de gás presente no mesmo e posterior manutenção da extremidade da agulha vedada com rolha de borracha, antes do procedimento de homogeneização. Aferiu-se a temperatura retal de cada bovino estudado anteriormente ao início do trabalho de colheita das amostras.

\section{Manutenção das amostras}

Ao término da colheita, o par de seringas foi transferido imediatamente para o laboratório, sendo cada uma submetida a um dos critérios distintos de manutenção ao longo das 24 horas que compreenderam o acompanhamento experimental. A amostra não conservada foi mantida à temperatura ambiente, registrando-se 23 e $30^{\circ} \mathrm{C}$ como mínima e máxima, respectivamente, no período, e, o material conservado permaneceu, por sua vez, entre zero e $4^{\circ} \mathrm{C}$, com a seringa submersa na água gelada.

Este método de conservação constituiu-se no emprego de caixa térmica de poliestireno expandido (Isopor ${ }^{\circledR}$ ) com capacidade para 7 litros (dimensões internas de $25 \times 17 \times 19,6 \mathrm{~cm}$ ), acondicionando 2 litros de água e $2 \mathrm{~kg}$ de gelo reciclável (gel de celulose) em embalagem plástica flexível.

Em respeito à necessidade de que a temperatura da água não chegasse a exceder o limite superior de $4^{\circ} \mathrm{C}$, a mesma foi aferida com constância durante o período experimental, efetuando-se a troca do gelo reciclável por quantidade igual à original na $12^{\mathrm{a}}$ hora. Tal proporção entre gelo e água, bem como a decisão do momento propício para a troca do gelo, basearam-se nas repetições dos resultados de experiências controladas realizadas previamente com essa finalidade.

\section{Processamento das amostras}

A dosagem da hemoglobina $(\mathrm{Hb})$, realizada no dia anterior ao do experimento, foi procedida 
pelo método clássico da cianometahemoglobina (BIRGEL, 1982), empregando-se reagente ${ }^{c}$ e padrão ${ }^{d}$ comerciais específicos com leitura por espectrofotometria ${ }^{\mathrm{e}}$.

Os exames hemogasométricos foram efetuados em todas as amostras nos momentos assim definidos: imediatamente após a colheita (com intervalo de, no máximo, 10 minutos) e com 1, 2, 3, 4, 5, $6,8,10,12$ e 24 horas transcorridas, utilizando-se o analisador automático de $\mathrm{pH}$ e gases sangüíneos ${ }^{\mathrm{f}}$, o qual possui a capacidade de mensurar o $\mathrm{pH}$ e as pressões parciais venosas de dióxido de carbono $\left(\mathrm{Pv}_{\mathrm{CO} 2}\right)$ e de oxigênio $\left(\mathrm{Pv}_{\mathrm{O} 2}\right)$ por meio de um sistema de eletrodos específicos (SEVERINGHAUS \& BRADLEY, 1958), e estabelecer automaticamente, segundo os critérios de SIGGAARD-ANDERSEN (1963), o cálculo das demais variáveis de interesse na avaliação do equilíbrio ácido-básico: concentração de bicarbonato no plasma $\left(\mathrm{HCO}_{3}{ }^{-}\right)$, total de dióxido de carbono no plasma $\left(\mathrm{TCO}_{2}\right)$, excesso ou déficit de bases no sangue (BE), concentração de bicarbonato padrão no sangue ( $\mathrm{StB})$, saturação de oxigênio no sangue $\left(\mathrm{SatO}_{2}\right)$ e conteúdo de oxigênio no sangue $\left(\mathrm{O}_{2}\right)$.

Deve-se destacar que, anteriormente à introdução da alíquota necessária no aparelho $(0,2 \mathrm{ml})$, realizava-se uma homogeneização adequada da amostra e desprezavam-se cerca de $0,5 \mathrm{ml}$ de sangue, sendo este o único e curto período em que a agulha era desacoplada da seringa, evitando-se ao máximo o contato com o ar. Ao término desse procedimento, eram fornecidas ao sistema de computador interno do hemogasômetro as informações do valor da $\mathrm{Hb}$ e da temperatura retal que o animal correspondente à amostra exibia pouco antes da colheita. Tais informações possuem o caráter de correção dos valores padrões assumidos pelo aparelho e referentes à espécie humana, a saber: $37^{\circ} \mathrm{C}$ e $15,0 \mathrm{~g} / \mathrm{dl}$, para a temperatura e $\mathrm{Hb}$, respectivamente. A temperatura corrigida causa influência nas determinações de $\mathrm{pH}, \mathrm{Pv}_{\mathrm{CO} 2}$ e $\mathrm{Pv}_{\mathrm{O} 2}$, e, esses valores medidos, juntamente com a $\mathrm{Hb}$ corrigida, são empregados no cálculo das demais variáveis estudadas.

\section{Análise estatística}

Os resultados foram analisados considerando dois experimentos em separado relativos a cada um dos critérios estabelecidos para a manutenção das amostras. Para cada caso, portanto, testaramse, inicialmente, os efeitos do tempo de conservação, do sexo, e a interação entre os mesmos, por meio da análise de variância, levando-se em conta um experimento com dois fatores. Por se tratarem de amostras dependentes, optou-se, finalmente, pela análise de variância de medidas repetidas, verificando-se o contraste entre a média observada em cada tempo estudado e aquela obtida imediatamente após a colheita, pelo método de Tukey, com o cálculo da diferença mínima significativa e uma probabilidade de erro de 5\% (ZAR, 1984).

\section{RESULTADOS E DISCUSSÃO}

Os animais estudados apresentaram temperatura retal oscilando entre 37,8 e $38,5^{\circ} \mathrm{C}(38,24 \pm$ $0,22^{\circ} \mathrm{C}$ ) e concentrações de $\mathrm{Hb}$ variando entre 8,0 e $11,3 \mathrm{~g} / \mathrm{dl}(9,95 \pm 0,91 \mathrm{~g} / \mathrm{dl})$, assim como valores hemogasométricos iniciais (Tabela 1) condizentes com o seu estado de higidez.

Conforme o resultado do primeiro tratamento estatístico, embora o efeito do tempo tenha sido significativo para a maioria das variáveis estudadas, não se verificou influência do sexo em qualquer uma delas nas amostras não conservadas ( $p>0,64)$ ou mantidas em água gelada $(p>0,62)$, e tão pouco interação entre esses dois fatores.

Os resultados obtidos encontram-se resumidos na tabela 1 e nas figuras 1 e 2 , demonstrando claramente o comportamento das variáveis estudadas ao longo do tempo nos dois métodos distintos empregados para a manutenção das amostras. Para maior facilidade de interpretação, optou-se, na tabela 1, pela apresentação de valores que expressam a diferença entre a média observada em cada tempo e aquela do momento imediato à colheita do material.

Analisando-se os valores médios iniciais (Tabela 1), é possível detectar uma certa diferença entre ambas as amostras, a qual ocorreu com maior ou menor magnitude para todas as variáveis estudadas, e, como documentado por HASKINS (1977), deve-se, provavelmente, ao erro intrínseco de colheita e manipulação do material, não tendo ultrapassado os limites de significância apontados por esse pesquisador.

Pode-se apreciar na tabela 1 e nas figuras 1 e 2, que, no material mantido à temperatura ambiente, as alterações ao longo do tempo caracterizaram-se por redução contínua do $\mathrm{pH}, \mathrm{Pv}_{\mathrm{O} 2}, \mathrm{BE}$ e $\mathrm{StB}$, e por elevação seqüencial da $\mathrm{Pv}_{\mathrm{CO} 2}$. Esse conjunto de modificações apresenta coerência com os resultados dos estudos de POULSEN \& SURYNEK (1977), ASSAL \& POULSEN (1978) e de ASSAL $\boldsymbol{e}$ t al. (1978; 1980), os quais, empregando métodos semelhantes aos do presente trabalho, tiveram como objetivo a compreensão das alterações que o sangue experimenta durante a estocagem, nas espécies bovina, eqüina, canina e suína, respectivamente. Todos esses autores demonstraram, ainda, uma elevação concomitante na concentração do ácido lático, in- 
Tabela 1 - Variação do $\mathrm{pH}$, pressões parciais de dióxido de carbono $\left(\mathrm{Pv}_{\mathrm{CO} 2}\right)$ e de oxigênio $\left(\mathrm{Pv}_{\mathrm{O} 2}\right)$, bicarbonato $\left(\mathrm{HCO}_{3}{ }^{-}\right)$, total de dióxido de carbono $\left(\mathrm{TCO}_{2}\right)$, excesso ou déficit de bases $(\mathrm{BE})$, bicarbonato padrão $(\mathrm{StB})$, saturação de oxigênio $\left(\mathrm{SatO}_{2}\right)$ e do conteúdo de oxigênio $\left(\mathrm{O}_{2}\right)$ em amostras de sangue venoso de bovinos $(\mathrm{n}=14)$ conservadas em banho de água gelada (zero a $\left.4^{\circ} \mathrm{C}\right)$ e não conservadas $\left(23\right.$ a $\left.30^{\circ} \mathrm{C}\right)$, expressa como a diferença entre a média de cada tempo transcorrido e aquela do momento da colheita.

\begin{tabular}{|c|c|c|c|c|c|c|c|c|c|c|c|c|}
\hline Variável & Manutenção & $\begin{array}{l}\text { Momento } \\
\text { da colheita }\end{array}$ & $1 \mathrm{~h}$ & $2 \mathrm{~h}$ & $3 \mathrm{~h}$ & $4 \mathrm{~h}$ & $5 \mathrm{~h}$ & $6 \mathrm{~h}$ & $8 \mathrm{~h}$ & $10 \mathrm{~h}$ & $12 \mathrm{~h}$ & $24 \mathrm{~h}$ \\
\hline $\mathrm{pH}$ & $\begin{array}{c}23 \text { a } 30^{\circ} \mathrm{C} \\
0 \text { a } 4^{\circ} \mathrm{C}\end{array}$ & $\begin{array}{l}7,397 \pm 0,026 \\
7,398 \pm 0,022\end{array}$ & $\begin{array}{l}-0,006 \\
-0,002\end{array}$ & $\begin{array}{l}-0,015^{*} \\
-0,005\end{array}$ & $\begin{array}{l}-0,022 * \\
-0,005\end{array}$ & $\begin{array}{l}-0,031 * \\
-0,006^{*}\end{array}$ & $\begin{array}{l}-0,037^{*} \\
-0,008^{*}\end{array}$ & $\begin{array}{l}-0,044^{*} \\
-0,011^{*}\end{array}$ & $\begin{array}{l}-0,057^{*} \\
-0,014^{*}\end{array}$ & $\begin{array}{l}-0,071^{*} \\
-0,017^{*}\end{array}$ & $\begin{array}{l}-0,080^{*} \\
-0,022^{*}\end{array}$ & $\begin{array}{l}-0,156^{*} \\
-0,032^{*}\end{array}$ \\
\hline $\begin{array}{l}\mathrm{Pv}_{\mathrm{CO} 2} \\
(\mathrm{mmHg})\end{array}$ & $\begin{array}{c}23 \text { a } 30^{\circ} \mathrm{C} \\
0 \text { a } 4^{\circ} \mathrm{C}\end{array}$ & $\begin{array}{l}41,84 \pm 3,01 \\
42,33 \pm 2,62\end{array}$ & $\begin{array}{l}0,84 \\
0,56\end{array}$ & $\begin{array}{l}1,41 \\
0,35\end{array}$ & $\begin{array}{l}2,34^{*} \\
0,52\end{array}$ & $\begin{array}{l}2,80^{*} \\
0,35\end{array}$ & $\begin{array}{l}3,68^{*} \\
0,54\end{array}$ & $\begin{array}{l}4,50^{*} \\
0,82^{*}\end{array}$ & $\begin{array}{l}5,74^{*} \\
1,00^{*}\end{array}$ & $\begin{array}{l}7,25^{*} \\
1,37^{*}\end{array}$ & $\begin{array}{l}8,64^{*} \\
1,93^{*}\end{array}$ & $\begin{array}{r}18,69^{*} \\
3,08^{*}\end{array}$ \\
\hline $\begin{array}{l}\mathrm{Pv}_{\mathrm{O} 2} \\
(\mathrm{mmHg})\end{array}$ & $\begin{array}{c}23 \text { a } 30^{\circ} \mathrm{C} \\
0 \text { a } 4^{\circ} \mathrm{C}\end{array}$ & $\begin{array}{l}35,81 \pm 5,81 \\
34,03 \pm 4,63\end{array}$ & $\begin{array}{r}-0,26 \\
0,13\end{array}$ & $\begin{array}{r}-0,60 \\
0,04\end{array}$ & $\begin{array}{r}-0,80 \\
0,01\end{array}$ & $\begin{array}{r}-1,09 \\
0,19\end{array}$ & $\begin{array}{r}-1,31 \\
0,30\end{array}$ & $\begin{array}{c}-1,73^{*} \\
0,36\end{array}$ & $\begin{array}{c}-2,22^{*} \\
0,43\end{array}$ & $\begin{array}{r}-2,81 * \\
0,49 *\end{array}$ & $\begin{array}{r}-3,31^{*} \\
0,65^{*}\end{array}$ & $\begin{array}{r}-5,16^{*} \\
1,40^{*}\end{array}$ \\
\hline $\begin{array}{l}\mathrm{HCO}_{3}^{-} \\
(\mathrm{mmol} / \mathrm{l})\end{array}$ & $\begin{array}{c}23 \text { a } 30^{\circ} \mathrm{C} \\
0 \text { a } 4^{\circ} \mathrm{C}\end{array}$ & $\begin{array}{l}25,05 \pm 1,94 \\
25,39 \pm 1,82\end{array}$ & $\begin{array}{l}0,12 \\
0,17\end{array}$ & $\begin{array}{l}-0,12 \\
-0,05\end{array}$ & $\begin{array}{r}0,00 \\
-0,01\end{array}$ & $\begin{array}{l}-0,27 \\
-0,19\end{array}$ & $\begin{array}{l}-0,11 \\
-0,16\end{array}$ & $\begin{array}{l}-0,14 \\
-0,21\end{array}$ & $\begin{array}{l}-0,21 \\
-0,24\end{array}$ & $\begin{array}{l}-0,33 \\
-0,16\end{array}$ & $\begin{array}{l}-0,19 \\
-0,20\end{array}$ & $\begin{array}{l}-0,36^{*} \\
-0,21\end{array}$ \\
\hline $\begin{array}{l}\mathrm{TCO}_{2} \\
(\mathrm{mmol} / \mathrm{l})\end{array}$ & $\begin{array}{c}23 \text { a } 30^{\circ} \mathrm{C} \\
0 \text { a } 4^{\circ} \mathrm{C}\end{array}$ & $\begin{array}{l}26,24 \pm 2,00 \\
26,61 \pm 1,86\end{array}$ & $\begin{array}{l}0,17 \\
0,19\end{array}$ & $\begin{array}{l}-0,07 \\
-0,06\end{array}$ & $\begin{array}{l}0,09 \\
0,01\end{array}$ & $\begin{array}{l}-0,19 \\
-0,19\end{array}$ & $\begin{array}{l}-0,01 \\
-0,16\end{array}$ & $\begin{array}{r}0,01 \\
-0,21\end{array}$ & $\begin{array}{l}-0,03 \\
-0,21\end{array}$ & $\begin{array}{l}-0,11 \\
-0,11\end{array}$ & $\begin{array}{r}0,08 \\
-0,16\end{array}$ & $\begin{array}{r}0,19 \\
-0,13\end{array}$ \\
\hline $\begin{array}{l}\mathrm{BE} \\
(\mathrm{mmol} / \mathrm{l})\end{array}$ & $\begin{array}{c}23 \text { a } 30^{\circ} \mathrm{C} \\
0 \text { a } 4^{\circ} \mathrm{C}\end{array}$ & $\begin{array}{l}0,76 \pm 1,88 \\
1,08 \pm 1,74\end{array}$ & $\begin{array}{r}-0,01 \\
0,09\end{array}$ & $\begin{array}{l}-0,36 \\
-0,14\end{array}$ & $\begin{array}{l}-0,39 \\
-0,10\end{array}$ & $\begin{array}{l}-0,78^{*} \\
-0,29\end{array}$ & $\begin{array}{l}-0,75^{*} \\
-0,29\end{array}$ & $\begin{array}{l}-0,91^{*} \\
-0,40^{*}\end{array}$ & $\begin{array}{l}-1,22^{*} \\
-0,45^{*}\end{array}$ & $\begin{array}{l}-1,61^{*} \\
-0,48^{*}\end{array}$ & $\begin{array}{l}-1,61^{*} \\
-0,56^{*}\end{array}$ & $\begin{array}{l}-3,26^{*} \\
-0,76^{*}\end{array}$ \\
\hline $\begin{array}{l}\text { StB } \\
(\mathrm{mmol} / \mathrm{l})\end{array}$ & $\begin{array}{c}23 \text { a } 30^{\circ} \mathrm{C} \\
0 \text { a } 4^{\circ} \mathrm{C}\end{array}$ & $\begin{array}{l}24,55 \pm 1,58 \\
24,78 \pm 1,41\end{array}$ & $\begin{array}{r}-0,01 \\
0,08\end{array}$ & $\begin{array}{l}-0,36 \\
-0,14\end{array}$ & $\begin{array}{l}-0,39^{*} \\
-0,10\end{array}$ & $\begin{array}{l}-0,74 * \\
-0,27\end{array}$ & $\begin{array}{l}-0,74 * \\
-0,25\end{array}$ & $\begin{array}{l}-0,89^{*} \\
-0,36^{*}\end{array}$ & $\begin{array}{l}-1,16^{*} \\
-0,41^{*}\end{array}$ & $\begin{array}{l}-1,54 * \\
-0,41^{*}\end{array}$ & $\begin{array}{l}-1,58^{*} \\
-0,50^{*}\end{array}$ & $\begin{array}{l}-3,04 * \\
-0,67 *\end{array}$ \\
\hline $\mathrm{SatO}_{2}(\%)$ & $\begin{array}{c}23 \text { a } 30^{\circ} \mathrm{C} \\
0 \text { a } 4^{\circ} \mathrm{C}\end{array}$ & $\begin{array}{c}63,09 \pm 10,41 \\
60,75 \pm 9,30\end{array}$ & $\begin{array}{r}-1,03 \\
0,35\end{array}$ & $\begin{array}{r}-2,24 \\
0,04\end{array}$ & $\begin{array}{c}-3,07 * \\
0,00\end{array}$ & $\begin{array}{c}-4,22^{*} \\
0,23\end{array}$ & $\begin{array}{c}-5,04 * \\
0,29\end{array}$ & $\begin{array}{c}-6,37 * \\
0,23\end{array}$ & $\begin{array}{c}-8,28 * \\
0,16\end{array}$ & $\begin{array}{c}-10,50 * \\
-0,09\end{array}$ & $\begin{array}{c}-12,03 * \\
-0,21\end{array}$ & $\begin{array}{c}-20,61 * \\
0,67\end{array}$ \\
\hline $\mathrm{O}_{2}(\mathrm{vol} \%)$ & $\begin{array}{c}23 \text { a } 30^{\circ} \mathrm{C} \\
0 \text { a } 4^{\circ} \mathrm{C}\end{array}$ & $\begin{array}{l}8,88 \pm 2,00 \\
8,41 \pm 1,64\end{array}$ & $\begin{array}{r}-0,15 \\
0,06\end{array}$ & $\begin{array}{r}-0,31 \\
0,01\end{array}$ & $\begin{array}{l}-0,44^{*} \\
-0,01\end{array}$ & $\begin{array}{c}-0,59^{*} \\
0,03\end{array}$ & $\begin{array}{c}-0,69^{*} \\
0,03\end{array}$ & $\begin{array}{c}-0,89^{*} \\
0,03\end{array}$ & $\begin{array}{c}-1,14 * \\
0,02\end{array}$ & $\begin{array}{c}-1,44^{*} \\
0,00\end{array}$ & $\begin{array}{l}-1,65^{*} \\
-0,01\end{array}$ & $\begin{array}{c}-2,82 * \\
0,12\end{array}$ \\
\hline
\end{tabular}

(*) $\mathrm{p}<0,05$ para o contraste com a média do momento da colheita.

dicando a continuidade da atividade metabólica das células sangüíneas, representada principalmente pelo mecanismo glicolítico anaeróbico, o qual se processa no interior dos eritrócitos. O acúmulo continuado dos ácidos lático e pirúvico como produtos finais dessa via metabólica, pode, portanto, justificar as alterações observadas "in vitro" nos parâmetros ácido-básicos.

Com exceção do aumento na $\mathrm{Pv}_{\mathrm{O} 2}$, a mesma tendência de comportamento dessas variáveis foi observada nas amostras conservadas, embora muito mais tardiamente do que no sangue não conservado (Tabela 1 e Figuras 1 e 2). Esses achados se assemelham aos citados na literatura (POULSEN \& SURYNEK, 1977; ASSAL \& POULSEN, 1978; ASSAL et al., 1978 e 1980) e reforçam a evidência de que a manutenção do material em temperaturas próximas a zero é capaz de retardar substancialmente as alterações ácido-básicas naturais esperadas, certamente em razão da inibição do metabolismo eritrocitário.
Os resultados apresentados para o conjunto das variáveis hemogasométricas no sangue conservado (Tabela 1) foram, uma vez mais, compatíveis com aqueles apontados para amostras de sangue venoso bovino conservadas em água gelada entre zero e $3^{\circ} \mathrm{C}$ (JAGOS et al., 1977) ou em refrigerador a $4^{\circ} \mathrm{C}$ (SZENCI \& BESSER, 1990; SZENCI et al., 1991), incluindo, nos dois últimos estudos, a elevação da $\mathrm{Pv}_{\mathrm{O} 2}$ e a ausência de variações estatisticamente significativas para $\mathrm{HCO}_{3}^{-}, \mathrm{TCO}_{2}$ e $\mathrm{SatO}_{2}$ com o decorrer do tempo.

Guardadas as similaridades mencionadas, deve-se destacar, contudo, a existência de uma discrepância relativa com respeito ao momento transcorrido desde a colheita, a partir do qual cada variável hemogasométrica passou a assumir valor significativamente diferente daquele que exibia originalmente. Neste particular, como indicado na tabela 1, os momentos críticos estabelecidos foram: 4 horas para o $\mathrm{pH}, 6$ horas para a $\mathrm{Pv}_{\mathrm{CO} 2}$, $\mathrm{BE}$ e $\mathrm{StB}$, e 10 horas para a $\mathrm{Pv}_{\mathrm{O} 2}$; sendo que JAGOS et al. (1977)

Ciência Rural, v. 31, n. 2, 2001. 


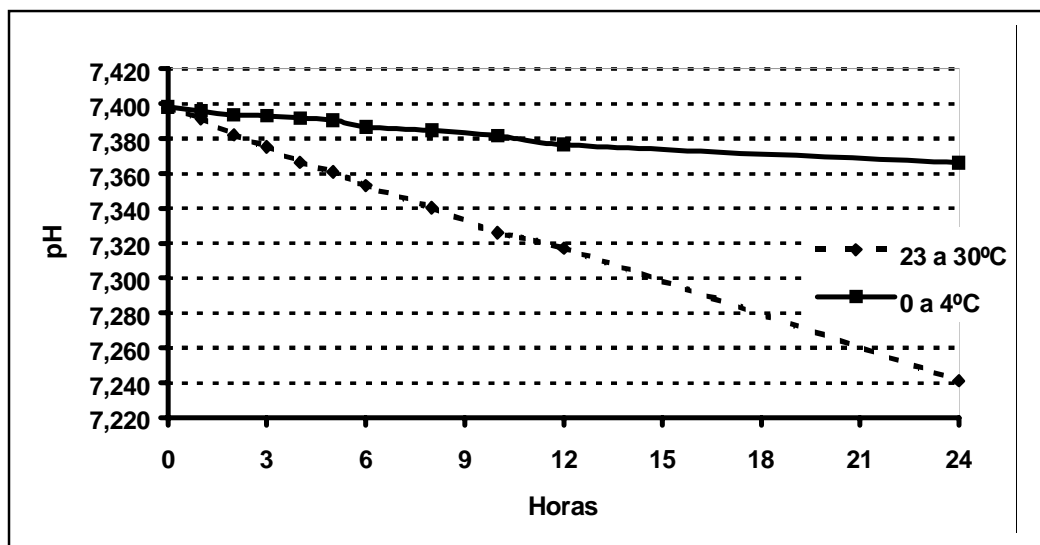

Figura 1 - Perfil do pH nas amostras de sangue venoso bovino $(\mathrm{n}=14)$ mantidas à temperatura ambiente $\left(23\right.$ a $\left.30^{\circ} \mathrm{C}\right)$ ou conservadas em banho de água gelada $\left(0\right.$ a $\left.4^{\circ} \mathrm{C}\right)$, ao longo do período de 24 horas que sucedeu a colheita.

referiram o tempo de 5 horas pós-colheita para o $\mathrm{pH}$, $\mathrm{Pv}_{\mathrm{CO} 2}$, BE e StB. No seu estudo, SZENCI \& BESSER (1990) obtiveram variação significativa já na terceira hora de conservação para o $\mathrm{pH}, \mathrm{Pv}_{\mathrm{O} 2}, \mathrm{BE}$ e $\mathrm{StB}$, e somente a partir de 9 horas para a $\mathrm{Pv}_{\mathrm{CO} 2}$. Essa diversidade nos resultados poderia ser considerada como um provável reflexo das diferenças entre os métodos empregados em cada um dos experimentos.

Apesar das evidências referidas, JAGOS et al. (1977) e SZENCI \& BESSER (1990) concluíram que, do ponto de vista prático, o tempo de viabilidade das amostras conservadas de sangue venoso bovino poderia ser aceito, respectivamente, como de 8 ou até mesmo 24 horas. Tais conclusões apoiaramse na observação de que os valores médios reais de cada tempo não se distanciavam muito dos obtidos no momento da colheita. Dessa forma, a interpretação do exame hemogasométrico continuaria a pos-

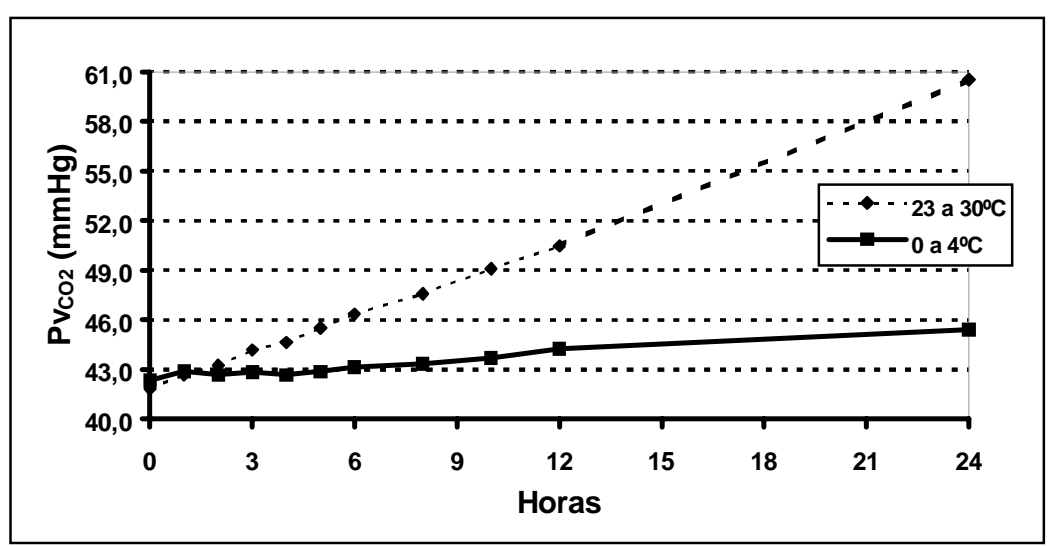

Figura 2 - Perfil da pressão parcial de dióxido de carbono $\left(\mathrm{Pv}_{\mathrm{CO} 2}\right)$ nas amostras de sangue venoso bovino $(\mathrm{n}=14)$ mantidas à temperatura ambiente $\left(23\right.$ a $\left.30^{\circ} \mathrm{C}\right)$ ou conservadas em banho de água gelada $\left(0\right.$ a $\left.4^{\circ} \mathrm{C}\right)$, ao longo do período de 24 horas que sucedeu a colheita. suir valor diagnóstico, contrariando o conceito clássico de realizar a determinação o mais rápido possível após a colheita (COLES, 1984). Com base nos resultados ora estabelecidos (Tabela 1), parece razoável, no entanto, indicar o período de 6 horas transcorridas desde a colheita da amostra como o limite máximo aceitável para a análise dos gases sanguiíneos e dos parâmetros ácido-básicos.

Naturalmente, essa conclusão não deve ser extrapolada para outras espécies, pois, conforme demonstrações anteriores (HASKINS, 1977; ASSAL et al., 1980; SZENCI et al., 1991), a velocidade com que as alterações "in vitro" se estabelecem no sangue venoso conservado varia de forma substancial entre as espécies, obedecendo, em geral, a uma ordem decrescente para o cão, o eqüino, o suíno e o bovino.

Cabe salientar por fim, que o método utilizado para conservação mostrou-se eficiente ao cumprir o seu propósito, e apresenta a simplicidade e a pronta aplicação na prática como vantagens adicionais, em razão do material necessário ser de fácil aquisição e de baixo custo. Para o profissional que atua no campo, o intervalo de tempo de viabilidade é amplo o suficiente para permitir o transporte do sangue, adequadamente conservado, até o laboratório mais acessível, assegurando a confiabilidade nos resultados da avaliação hemogasométrica, exame imprescindível para a interpretação dos desequilíbrios ácido-básicos presentes em certas enfermidades dos bovinos (BLOOD \& RADOSTITS, 1989; GONÇALVES et al., 1991; BENESI, 1993).

\section{CONCLUSÕES}

Nas condições em que se desenvolveu o experimento, a análise dos resultados permitiu concluir que as amostras de sangue venoso bovino destinadas ao exame hemogasométrico podem ser conservadas adequadamente, quando mantidas em banho de água gelada entre zero e $4^{\circ} \mathrm{C}$, fornecendo resultados confiáveis por até 6 horas após a sua colheita, o que gera oportunidade de sua utilização pelo clínico veterinário com atuação no campo.

Ciência Rural, v. 31, n. 2, 2001. 


\section{FONTES DE AQUISIÇÃO}

${ }^{a}$ Vacutainer, Becton Dikinson Indústrias Cirúrgicas Ltda.

${ }^{\mathrm{b}}$ Liquemine, ROCHE Químicos e Farmacêuticos S.A.

${ }^{\mathrm{c}}$ Reativo para hemoglobina, Labtest Diagnóstica S.A.

${ }^{\mathrm{d}}$ Padrão de hemoglobina, Labtest Diagnóstica S.A.

${ }^{\mathrm{e}}$ Espectrofotômetro E-225 D, CELM.

${ }^{\mathrm{f}}$ Hemogasômetro ABL 330, Radiometer, Copenhagen.

\section{REFERÊNCIAS BIBLIOGRÁFICAS}

ASSAL, A.N., ARNBJERG, J., POULSEN, J.S.D. Acid-base statuts of canine blood during storage. Nordisk Veterinaermedicin, v.30, p.345-353, 1978.

ASSAL, A.N., CHRISTIANSEN, I.J., POULSEN, J.S.D. Acidbase statuts of porcine blood during storage. Nordisk Veterinaermedicin, v.32, p.9-16, 1980.

ASSAL, A.N., POULSEN, J.S.D. Acid-base statuts of equine blood during storage. Nordisk Veterinaermedicin, v.30, p.354-363, 1978 .

BENESI, F.J. Síndrome asfixia neonatal dos bezerros. Importância e avaliação crítica. Arquivos da Escola de Medicina Veterinária da Universidade Federal da Bahia, v.16, p.38-48, 1993.

BIRGEL, E.H. Hematologia clínica veterinária. In: BIRGEL, E.H., BENESI, F.J. Patologia clínica veterinária. São Paulo : Sociedade Paulista de Medicina Veterinária, 1982. p. $2-50$.

BLOOD, D.C., RADOSTITS, O.M. Veterinary medicine. 7.ed London : Baillière Tindall, 1989. 1263p.

COLES, E.H. Patologia clínica veterinária. 3.ed. São Paulo : Manole, 1984. 566p.

GONÇALVES, R.C., KUCHEMBUCK, M.R.G., ALMEIDA,
C.T., et al. Diarréia em bezerros: estudo clínico e laboratorial. Veterinária e Zootecnia, v.3, p.35-44, 1991.

HASKINS, S.C. S ampling and storage of blood for $\mathrm{pH}$ and blood gas analysis. Journal of the American Veterinary Medical Association, v.170, p.429-433, 1977.

JAGOS, P., BOUDA, J., PRIKRYLOVA, J. The dynamics of the acid-base changes of bovine venous blood in vitro, as depending on time. Veterinární Medicína, v.22, p.257-262, 1977.

POULSEN, J.S.D., SURYNEK, J. Acid-base status of cattle blood. Sampling and storing. Nordisk Veterinaermedicin, v.29, p.271-283, 1977.

SEVERINGHAUS, J.W., BRADLEY, A.F. Electrodes for $\mathrm{pO}_{2}$ and $\mathrm{pCO}_{2}$ determination. Annales of Applyed Physiology, v.13, p.515-520, 1958.

SIGGAARD-ANDERSEN, O. Blood acid-base alignment nomogram. Scales for $\mathrm{pH}, \mathrm{pCO}_{2}$, base excess of whole blood of different hemoglobin concentrations, plasma bicarbonate, and plasma total $\mathrm{CO}_{2}$. Scandinavian Journal of Clinical Laboratory Investigation, v.15, p.211-220, 1963.

SZENCI, O., BESSER, T. Changes in blood gas and acid-base values of bovine venous blood during storage. Journal of the American Veterinary Medical Association, v.197, p.471-474, 1990 .

SZENCI, O., BRYDL, E., BAJCSY, C.A. Effect of storage on measurement of ionized calcium and acid-base variables in equine, bovine, ovine, and canine venous blood. Journal of the American Veterinary Medical Association, v.199, p.1167-1169, 1991.

ZAR, J.H. Biostatistical Analysis. 2. ed. Englewood Cliffs : Prentice-Hall, 1984. 718p.

Ciência Rural, v. 31, n. 2, 2001. 